\title{
Review Article \\ Clinical Significance of UCA1 to Predict Metastasis and Poor Prognosis of Digestive System Malignancies: A Meta-Analysis
}

\author{
Xiao-Dong Sun, ${ }^{1}$ Chen Huan, ${ }^{2}$ Wei Qiu, ${ }^{1}$ Da-Wei Sun, ${ }^{1}$ Xiao-Ju Shi, ${ }^{1}$ Chuan-Lei Wang, \\ Chao Jiang, ${ }^{1}$ Guang-Yi Wang, ${ }^{1}$ and Guo-Yue $\mathrm{Lv}^{1}$ \\ ${ }^{1}$ Department of Hepatobiliary and Pancreatic Surgery, The First Hospital of Jilin University, Changchun, Jilin Province 130021, China \\ ${ }^{2}$ Institute of Virology and AIDS Research, The First Hospital of Jilin University, Changchun, Jilin Province 130021, China
}

Correspondence should be addressed to Guang-Yi Wang; wgymd@sina.com and Guo-Yue Lv; lvguoyue@sina.com

Received 5 June 2016; Accepted 24 October 2016

Academic Editor: Stephen Fink

Copyright (C) 2016 Xiao-Dong Sun et al. This is an open access article distributed under the Creative Commons Attribution License, which permits unrestricted use, distribution, and reproduction in any medium, provided the original work is properly cited.

Purpose. Urothelial carcinoma-associated 1 (UCA1) has been reported to be overexpressed and correlated with progression in various cancers. However, the association between UCA1 expression and some clinicopathological features of digestive system malignancies, such as metastasis and survival, remains inconclusive. Therefore, a meta-analysis was performed to investigate the clinical significance of UCA1 in digestive system malignancies. Methods. Relevant literatures were searched in PubMed, Web of Science, Cochrane Library, and Embase databases updated to May 2016. Results. A total of 1089 patients from 10 studies were included in this meta-analysis. Meta-analysis results showed that digestive system malignancy patients with UCA1 overexpression were significantly more susceptible to developing lymph node metastasis (LNM) (OR = 1.85, 95\% CI: 1.28-2.67) and distant metastasis $(\mathrm{DM})(\mathrm{OR}=3.14,95 \% \mathrm{CI}$ : 1.77-5.58) and suffer from poor overall survival (OS) (HR = 2.31, 95\% CI: 1.89-2.82, univariate analysis; $\mathrm{HR}=2.24,95 \% \mathrm{CI}: 1.69-2.98$, multivariate analysis) and poor disease-free survival (DFS) (HR = 2.65, 95\% CI: 1.59 4.43, univariate analysis; $\mathrm{HR}=2.50,95 \% \mathrm{CI}: 1.62-3.86$, multivariate analysis). Conclusion. UCA1 overexpression was correlated with LNM, DM, poor OS, and poor DFS. UCA1 may serve as an indicator for metastasis and poor prognosis in digestive system malignancies.

\section{Introduction}

Digestive system malignancies have threatened human health seriously. According to the GLOBOCAN estimates, there were about 3.4 million new cases and 2.9 million deaths caused by digestive system malignancies in 2012 worldwide [1]. Although great achievements have been made in therapeutic approaches, such as surgery and chemotherapy, the outcome of digestive system malignancies remains poor. Nowadays, advantage of biomarkers in diagnosis and prognosis of cancers has been suggested, which might provide more precise information for individualized treatment and disease monitoring $[2,3]$.

Long noncoding RNAs (lncRNAs) are a class of RNAs longer than $200 \mathrm{nt}$ that lack protein-coding capacity [4]. Despite the fact that they used to be regarded as "junk" of genome, increasing number of studies have suggested the contribution of lncRNAs to various biological processes via transcriptional and posttranscriptional regulation $[5,6]$. In particular, role of lncRNA in carcinogenesis has been highlighted recently [7]. LncRNAs were found to be dysregulated and function as oncogene or tumor suppressor in various cancers. Furthermore, a growing body of evidence has demonstrated that there was significant association between the expression of IncRNA and the progression of cancer, including clinical-pathological features and survival, indicating that lncRNA can serve as biomarker for cancers. Some lncRNAs, such as HOX transcript antisense RNA (HOTAIR) and metastasis associated lung adenocarcinoma transcript 1 (MALAT1), have been illustrated to potentially predict metastasis and prognosis of digestive system malignancies through meta-analysis [8-10].

Recently, IncRNA urothelial carcinoma-associated 1 (UCA1) has attracted great attention due to its involvement 
in diverse cancers. UCA1, which was also called cancer upregulated drug resistant (CUDR), was originally identified in bladder transitional cell carcinoma in 2008 and suggested to promote cell proliferation and transformation [11, 12]. So far, the overexpression of UCA1 has been reported in other cancers, especially in digestive system malignancies, including hepatocellular carcinoma $[13,14]$, gastric cancer $[15,16]$, colorectal cancer [17-20], pancreatic cancer [21], and esophageal squamous cell carcinoma [22]. Although the association between UCA1 expression and cancer progression was a particular concern for these studies, these results were limited by small sample sizes or inconsistent conclusions. For instance, the patients with high UCA1 level in cancerous tissues were suggested to suffer from elevated lymph node metastasis (LNM) and distant metastasis (DM) rate in numerous cancers $[13,18,19,22]$; nevertheless, this association has not been detected in other studies $[15,17]$. Moreover, the issue of whether the overexpression of UCA1 could predict poor prognosis $[16,20]$ or not $[14]$ also needs to be clarified. Therefore, to investigate the clinical value of UCA1, we performed this quantitative meta-analysis to assess the correlation of UCA1 expression with metastasis and prognosis in digestive system malignancies.

\section{Material and Methods}

2.1. Literature Search Strategy. All literatures investigating the association of lncRNA UCAl with metastasis and prognosis of digestive system malignancies were searched in PubMed, Web of Science, Cochrane Library, and Embase databases updated to May 2016. Search terms are as follows: "UCA1" or "urothelial carcinoma-associated 1" or "CUDR" or "cancer up-regulated drug resistant", "cancer" or "carcinoma" or "tumor" or "neoplasm", and "survival" or "prognosis" or "prognostic" or "progression" or "recurrence" or "outcome" or "metastasis" or "clinicopathological". The searching strategy used in PubMed was "(((((UCA1 [Title/Abstract]) OR urothelial carcinoma-associated 1 [Title/Abstract]) OR CUDR [Title/Abstract]) OR cancer up-regulated drug resistant [Title/Abstract] $))$ AND (((cancer) OR carcinoma) OR tumor) OR neoplasma)) AND $((((((($ survival) $)$ OR prognosis) OR prognositic) OR progression) OR recurrence) OR outcome) OR metastasis) OR clinicopathological)". In addition, the references in retrieved articles were screened manually for potential relevant studies.

2.2. Selection and Exclusion Criteria. The articles collected were considered eligible if they met the inclusion criteria: (1) articles were investigating the association of UCA1 with progression of digestive system malignancies; (2) the expression levels of UCA1 in primary cancerous tissues were measured; (3) patients were grouped according to the expression levels of UCA1; (4) related clinicopathological parameters were described. Exclusion criteria are the following: (1) duplicate publications; (2) reviews, letters, comments, and conference articles; (3) studies focusing on UCA1 in other types of cancers, rather than digestive system malignancies; (4) studies using cells lines or animals, rather than cancer patients; (5) studies without usable data. Regarding multiple publications from the same medical center, only the most recent or the most complete study was included in the meta-analysis.

2.3. Data Extract. Two investigators (Xiao-Dong Sun, Chen Huan) extracted data from the eligible studies independently, according to the inclusion and exclusion criteria. For disagreements, a consensus was achieved by a third investigator (Wei Qiu). The following information was collected from each eligible study: first author, publication year, region of patients, cancer type, total number of patients, detecting method of UCA1 expression, cut-off value of grouping, number of patients in high/low UCA1 expression group, number of patients with LNM and DM in each group, follow-up time, study endpoint, survival analysis method (multivariate or univariate), and hazard ratio (HR) with 95\% confidence interval (CI) for overall survival (OS) or disease-free survival (DFS). When the HRs and their 95\% CIs were given in the articles, these data were extracted directly. If the prognosis was plotted as Kaplan-Meier curve, data was digitized by the software Engauge Digitizer version 4.1 (http://digitizer.sourceforge.net/) and calculated as described [23].

2.4. Quality Assessment. Quality assessment of the included studies was performed according to the Newcastle-Ottawa Scale (NOS) criteria [24]. The NOS criteria is scored based on three aspects (subject selection, comparability of subject, and clinical outcome) with the final scores ranging from 0 to 9 , and a score $\geq 6$ indicates a high quality.

2.5. Data Analysis. To assess the heterogeneity among the included studies, $\chi^{2}$-based $Q$ test and $I^{2}$ statistics were used. When heterogeneity was significant $\left(I^{2}>50 \%\right.$ or $P<0.10$ for $\chi^{2}$ ), a random effects model was used; otherwise, fixed effects model was adopted. The potential publication bias was evaluated using a "funnel plot" as well as Begg's and Egger's test.

The meta-analysis was performed through using Stata SE12.0 (Stata Corporation). All $P$ values were two-sided, and $P<0.05$ was considered statistically significant.

\section{Results}

3.1. Literature Information. A total of 156 records were retrieved by searching the databases of PubMed, Web of Science, Cochrane Library, and Embase. After screening the title and abstract, 125 articles were excluded because they were either duplication or reviews or about other lncRNAs. For the thirty-one articles remaining, full text was assessed carefully and 21 were excluded for their insufficient data, or for they focused on the level of UCA1 from other cancers rather than digestive system malignancies. Finally, 10 articles comprising 1089 patients were identified as eligible and included in the present meta-analysis. The flow diagram was shown in Figure 1.

3.2. Study Characteristics. The baseline characteristics of the included studies were summarized in Table 1. The articles 


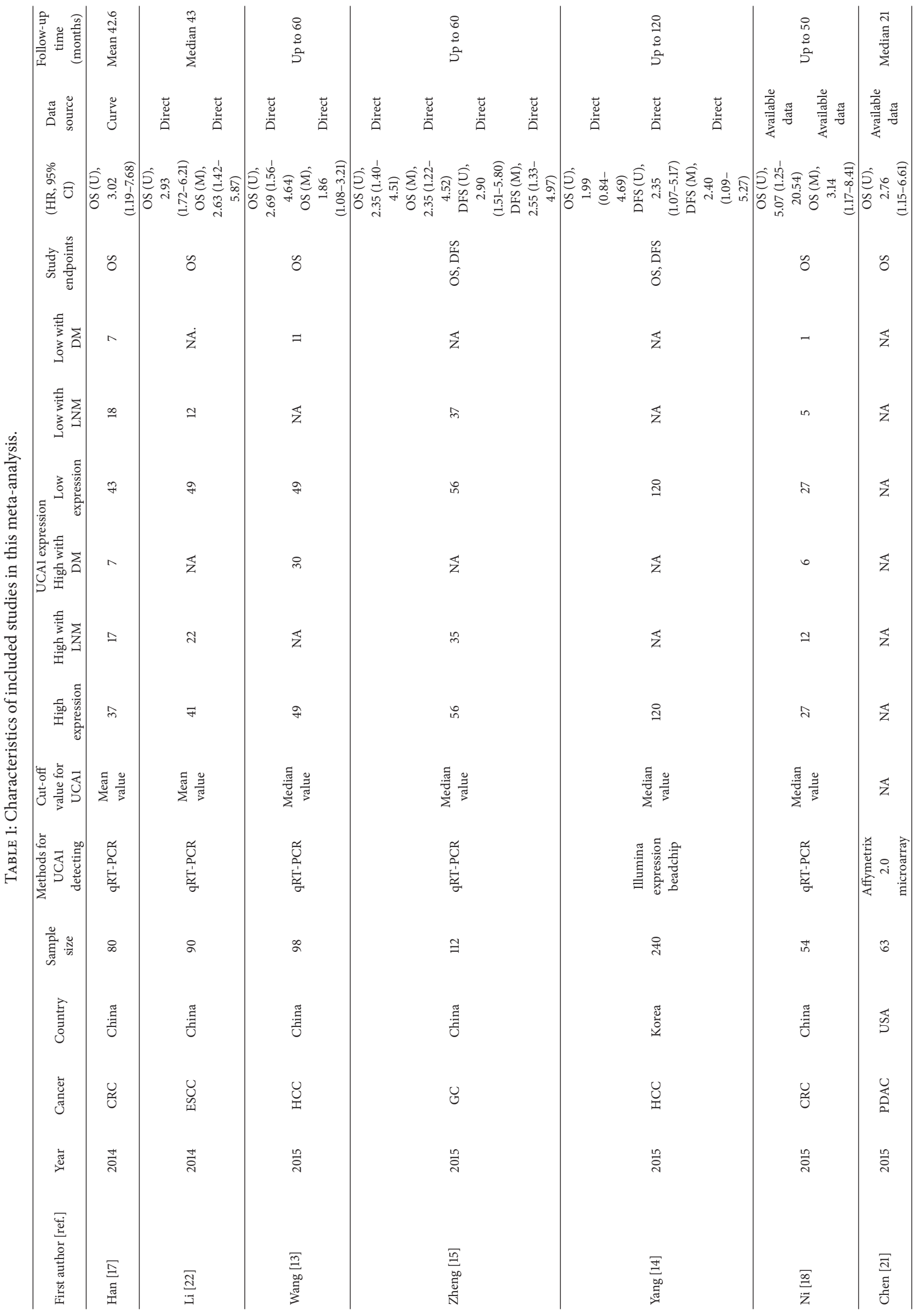




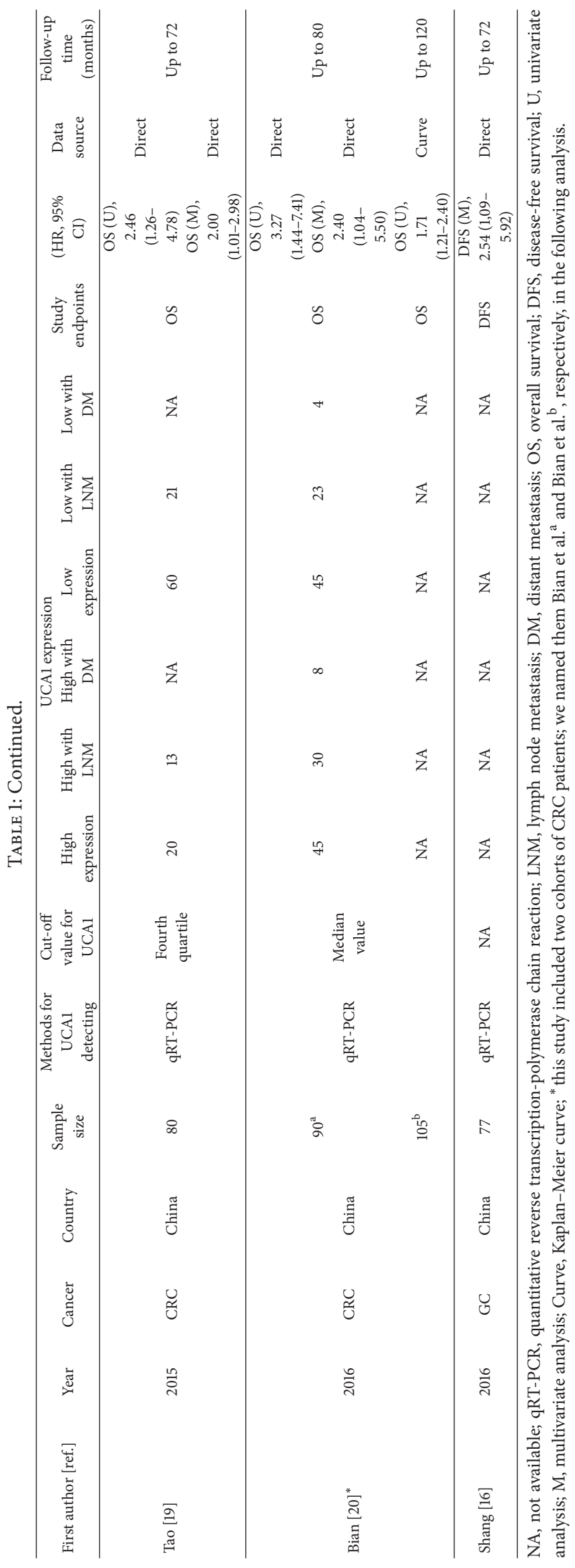




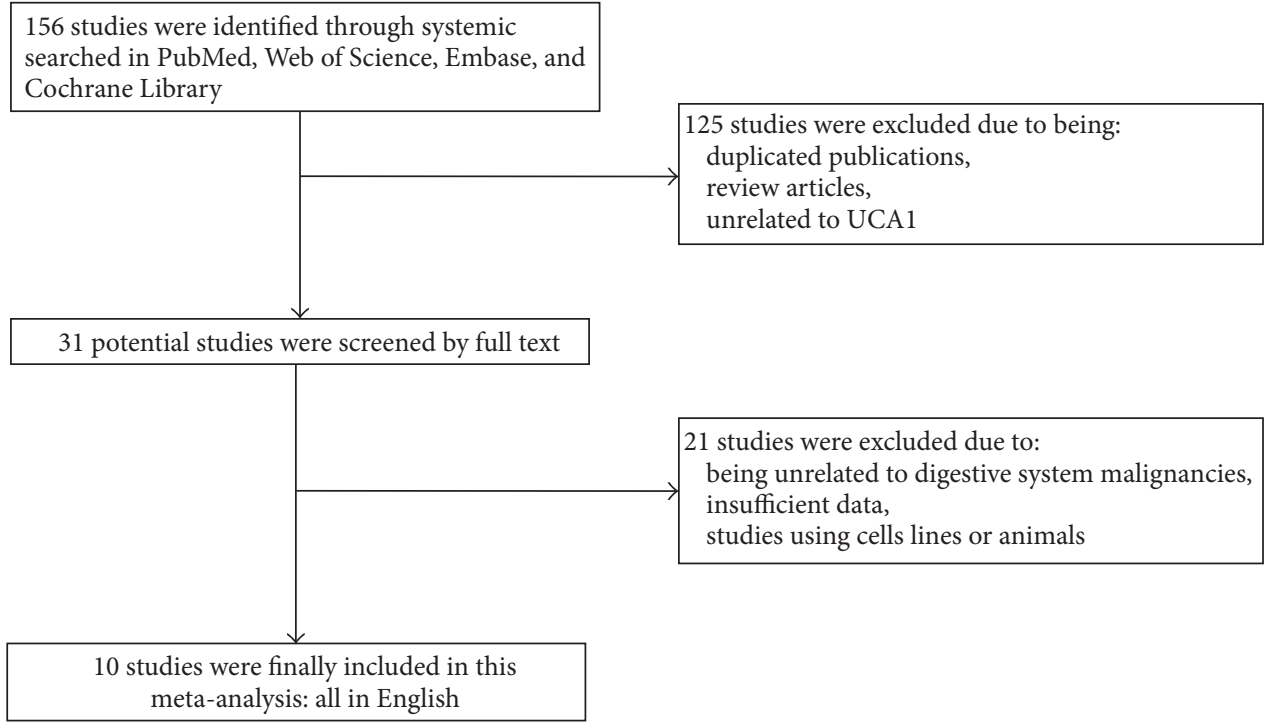

FIGURE 1: Flow diagram of searching relevant studies used in this meta-analysis.

were published between 2014 and 2016 with sample sizes ranging from 54 to 240 . Nearly all of them were conducted in Asia, 8 studies in China, 1 study in Korea, and 1 in USA. To detect the UCA1 expression, quantitative reverse transcription-polymerase chain reaction (RT-PCR) was used in 8 studies, Illumina expression beadchip was used in 1 study, and Affymetrix 2.0 microarray was used in 1 study. The cut-off value for UCA1 expression was unavailable in 2 studies, and the remaining 8 were based on median value of UCA1 level (in 5 studies), mean value of UCA1 level (in 2 studies), and fourth quartile of UCA1 level (in 1 study). According to the NOS criteria, all of the included studies got 7 scores or more, indicating their high methodological quality (Table 2).

\subsection{Meta-Analysis for OS}

3.3.1. Association between UCA1 and Metastasis. In total 6 studies including 506 cases reported the association of UCA1 with LNM of digestive system malignancies. Since there was no significant heterogeneity among these studies $\left(I^{2}=45.9 \%\right.$ and $P=0.100)$, the fixed model was adopted. The pooled OR with 95\% CI indicated that digestive system malignancy patients with high UCA1 level in tumor tissues were more susceptible to developing LNM (OR $=1.85$, 95\% CI: 1.28-2.67, $P=0.001$ ) (Figure 2(a)).

Moreover, there were four studies comprising 322 patients that investigated correlation of UCA1 expression and the occurrence of DM in digestive system malignancies. There was also no significant heterogeneity among these studies $\left(I^{2}=40.5 \%\right.$ and $\left.P=0.169\right)$, so the fixed model was applied to calculate the pooled OR and its 95\% CI. The result showed that increased UCA1 expression was significantly correlated with $\mathrm{DM}(\mathrm{OR}=3.14,95 \% \mathrm{CI}: 1.77-5.58, P=0.000)$ (Figure 2(b)).

Taken together, the results above showed that UCA1 overexpression was significantly correlated with LNM and
DM in digestive system cancer patients, suggesting that UCA1 may serve as an indicator for metastasis of digestive system malignancies.

3.3.2. Association between UCA1 and OS. On one hand, 9 studies with a total number of 1012 patients investigated the association between UCA1 expression and OS through univariate analysis. The fixed model was used to assess the pooled HR and its 95\% CI since no significant heterogeneity was found among these studies $\left(I^{2}=0 \%, P=0.707\right)$. We found that high UCA1 level was significantly associated with poor OS ( $\mathrm{HR}=2.31,95 \% \mathrm{CI}: 1.89-2.82, P=0.000)$ (Figure 3(a)). On the other hand, 6 studies with a total number of 524 patients investigated the association between UCA1 expression and OS through multivariate analysis. Since there was no significant heterogeneity among these studies $\left(I^{2}=0 \%, P=0.940\right)$, the fixed model was used to assess the pooled HR and its $95 \%$ CI. We found that UCA1 overexpression was also significantly associated with poor OS $(\mathrm{HR}=2.24,95 \% \mathrm{CI}: 1.69-2.98, P=0.000)$ (Figure 3(b)).

Results from the above analysis indicated that high expression of UCA1 was significantly correlated with poor OS in digestive system cancer patients, suggesting that UCA1 was an indicator of decreased survival rate in digestive system malignancies.

3.3.3. Association between UCA1 and DFS. Totally, there were 3 studies including 429 patients investigating the prognostic value of UCA1 on DFS in the form of either univariate or multivariate analysis. Since no significant heterogeneity was found among these studies $\left(I^{2}=0.0 \%, P=0.691\right.$, univariate analysis; $I^{2}=0.0 \%, P=0.992$, multivariate analysis), fixedeffect model was adopted to calculate the pooled HRs and their 95\% CIs. The results showed that increased UCA1 expression was also significantly associated with poor DFS 


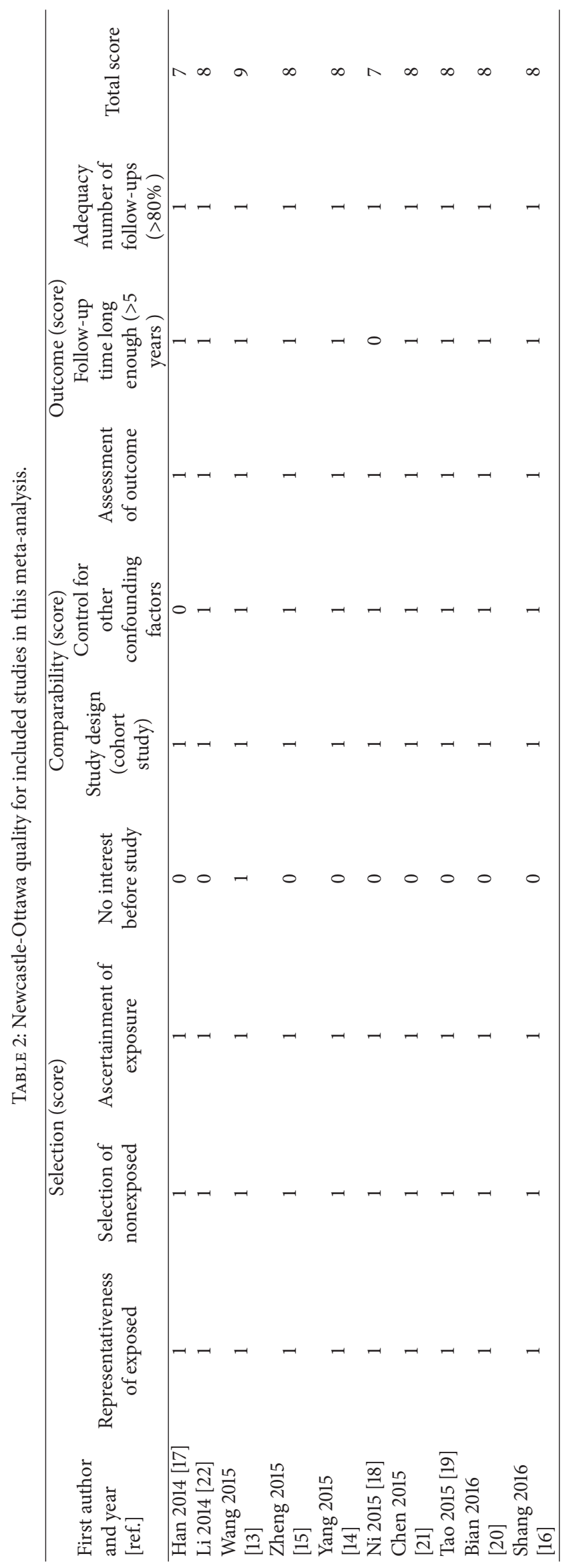




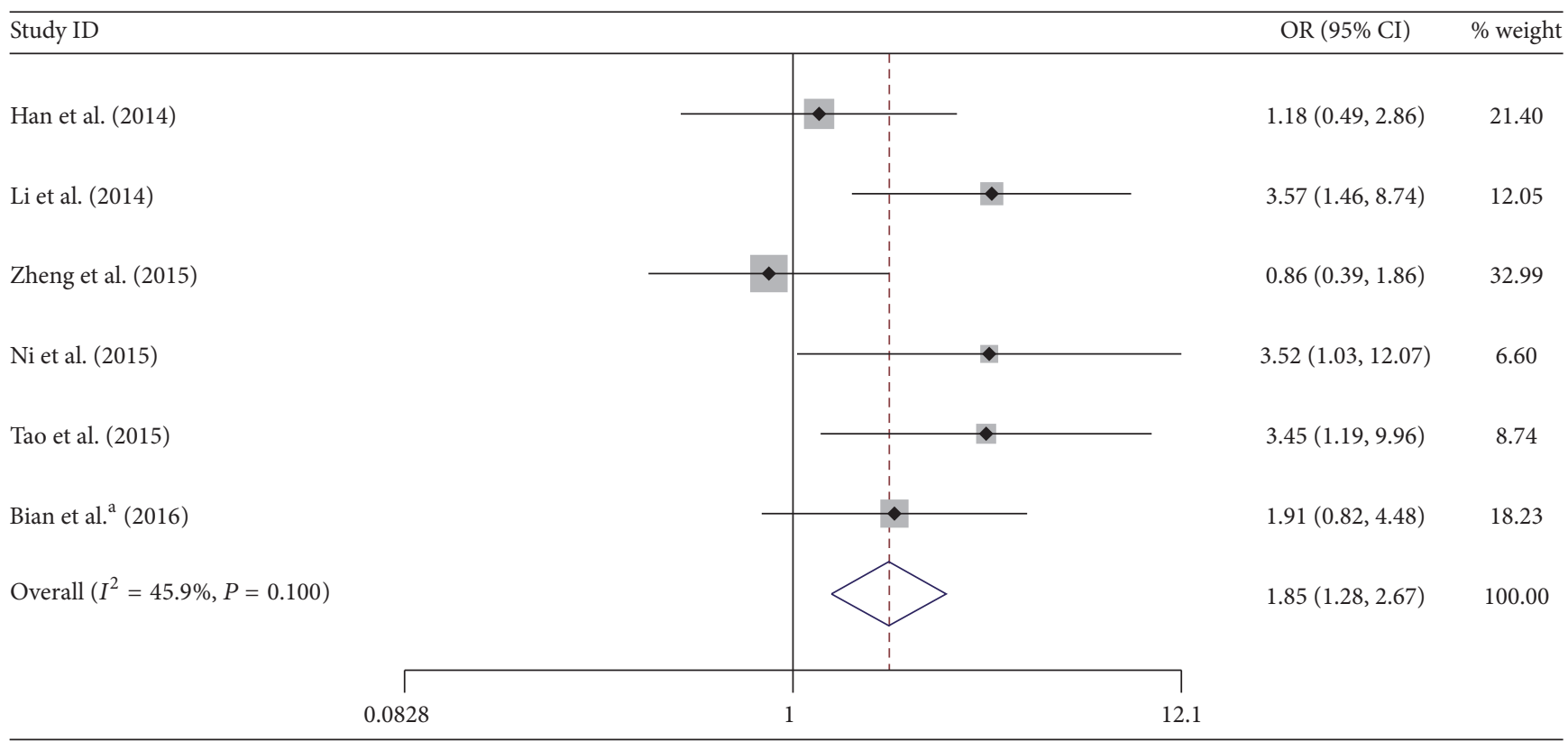

(a)

\begin{tabular}{|c|c|c|c|}
\hline Study ID & & OR (95\% CI) & $\%$ weight \\
\hline & i & & \\
\hline Han et al. (2014) & $\longrightarrow$ & $1.20(0.38,3.81)$ & 38.65 \\
\hline Wang et al. (2015) & $\frac{1}{1} \longrightarrow$ & $5.45(2.25,13.20)$ & 31.40 \\
\hline Ni et al. (2015) & 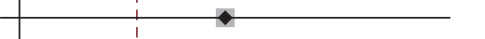 & $7.43(0.83,66.62)$ & 5.73 \\
\hline Bian et al. ${ }^{\mathrm{a}}$ (2016) & 1 & $2.22(0.62,7.97)$ & 24.22 \\
\hline Overall $\left(I^{2}=40.5 \%, P=0.169\right)$ & T. & $3.14(1.77,5.58)$ & 100.00 \\
\hline 0.015 & $\begin{array}{c}1 \\
66.6\end{array}$ & & \\
\hline
\end{tabular}

(b)

FIgURE 2: Forest plots of odds ratios (ORs) for the association between UCA1 expression and lymph node metastasis (LNM) (a) and distant metastasis (DM) (b).

$(\mathrm{HR}=2.65,95 \% \mathrm{CI}: 1.59-4.43, P=0.000$, univariate analysis; $\mathrm{HR}=2.50,95 \% \mathrm{CI}: 1.62-3.86, P=0.000$, multivariate analysis) (Figures 4(a) and 4(b)), indicating that increased UCA1 expression was an indicator of early tumor recurrence in digestive system cancer patients.

3.4. Publication Bias. In this meta-analysis, both Begg's and Egger's $P$ value tests were used to assess the potential publication bias. No publication bias was found in the studies with LNM $(P=0.188,0.109), \mathrm{DM}(P=1.000,0.949)$, or OS ( $P=0.128$ for Begg's test, univariate analysis $)$ or DFS $(P=$ $0.602,0.746$, multivariate analysis). However, publication bias was found in the studies with OS $(P=0.003$ for Egger's test, univariate analysis; $P=0.039,0.035$, multivariate analysis). Besides, the funnel plots for LNM (Figure 5(a)), DM (Figure 5(b)), OS from multivariate analysis (Figure 5(c)), and DFS from multivariate analysis (Figure 5(d)) were largely symmetrical. Therefore, we speculate that most of our metaanalysis results are reliable.

\section{Discussion}

Digestive system malignancies constitute a major part of human cancers [1]. Their rapid progression and poor outcome 


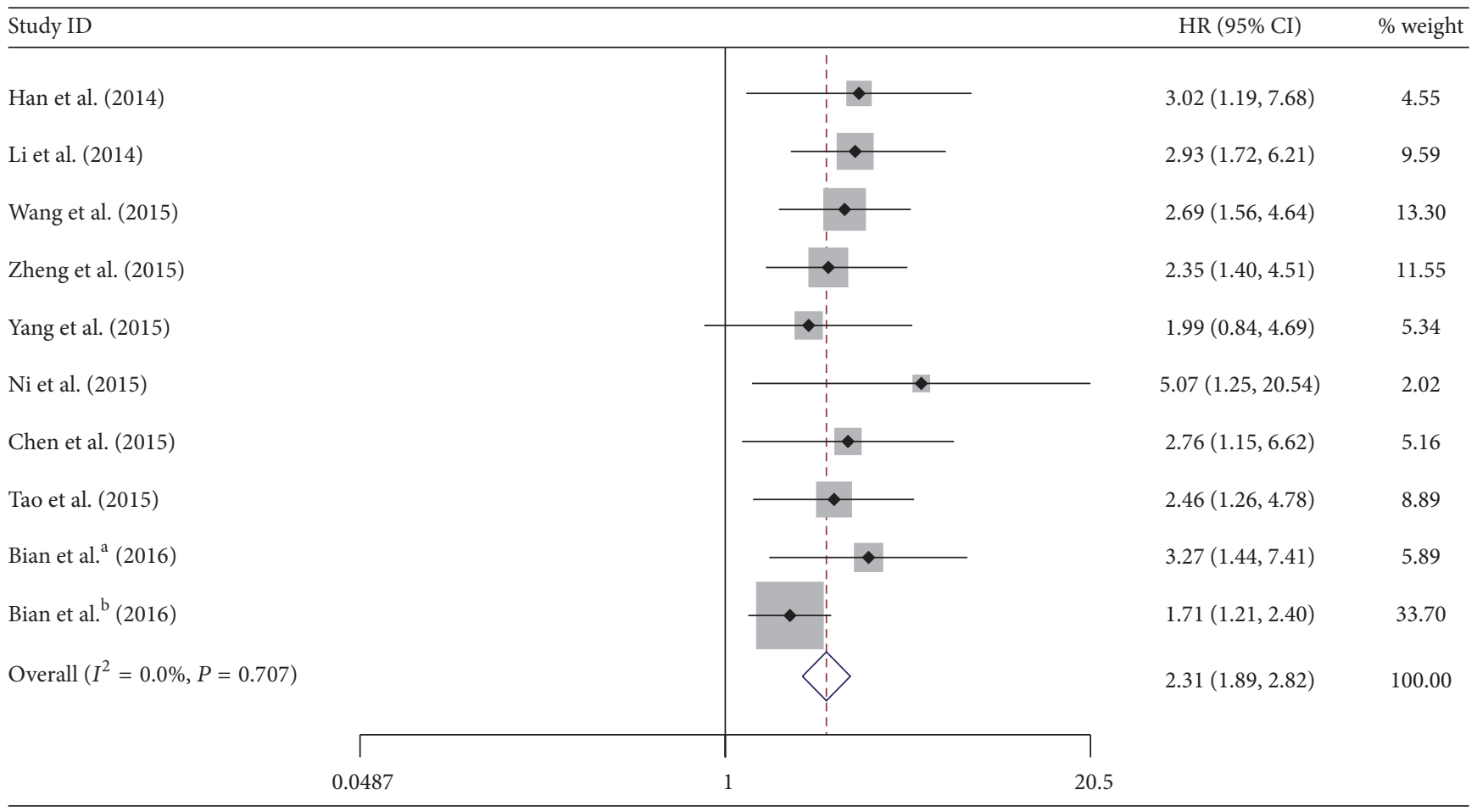

(a)

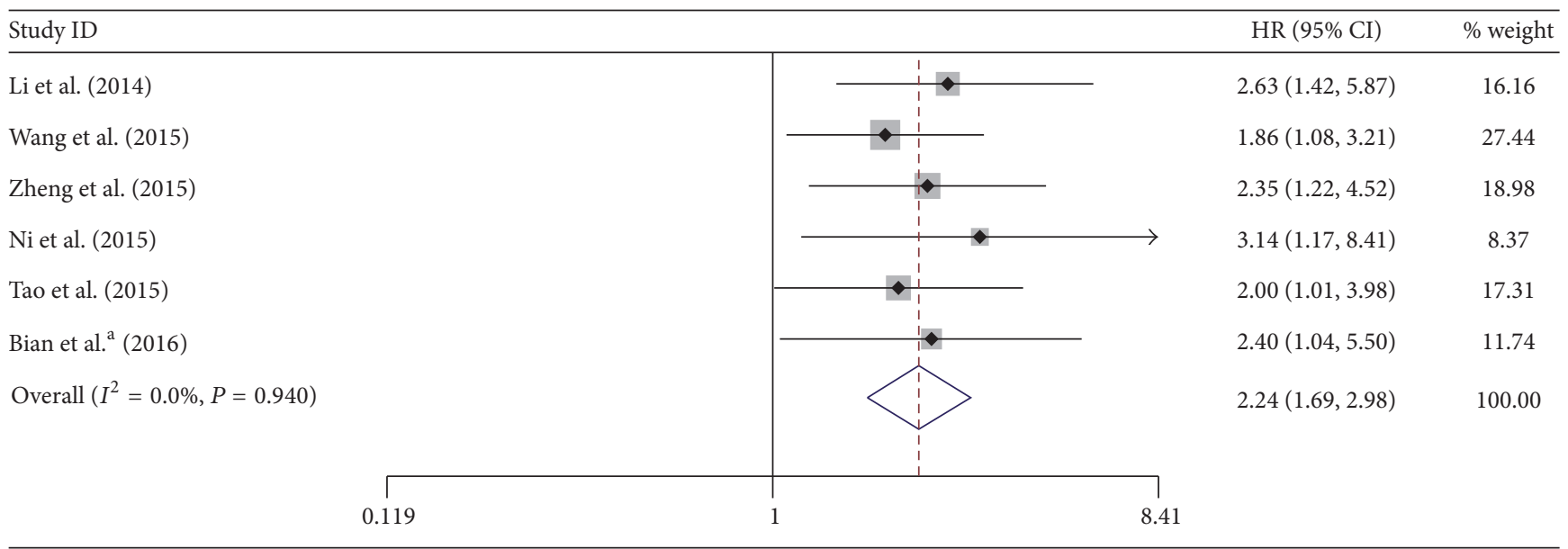

(b)

FIGURE 3: Forest plots of hazard ratios (HRs) for the association between UCA1 expression with overall survival (OS) from univariate analysis results (a) and OS from multivariate analysis results (b).

make it necessary and essential to identify biomarkers, which could improve the diagnosis and therapy by providing more precise and valuable information. UCA1, a lncRNA located at $19 \mathrm{p} 13.12$, has been found to be upregulated and exert oncogenic function in digestive system malignancies. In colorectal carcinoma, for example, overexpression of UCA1 was illustrated to promote proliferation and cell cycle progression and inhibit apoptosis, whereas suppression of UCA1 inhibited cell proliferation and cell cycle progression and facilitated apoptosis [17]. Regarding the mechanism, UCA1 can act as a competing endogenous RNA (ceRNA) by directly binding to microRNAs (miRNAs). In hepatocellular carcinoma, upregulated UCA1 contributes to the progression of cancer by counteracting the inhibitory effect of miR-216b and activating the FGFR1/ERK signaling pathway [13]. In colorectal cancer, UCA1 was found to function as an endogenous sponge by directly binding to miR-204-5p and promote the expression of a new target of miR-204-5p, CREB1 [20]. The results 


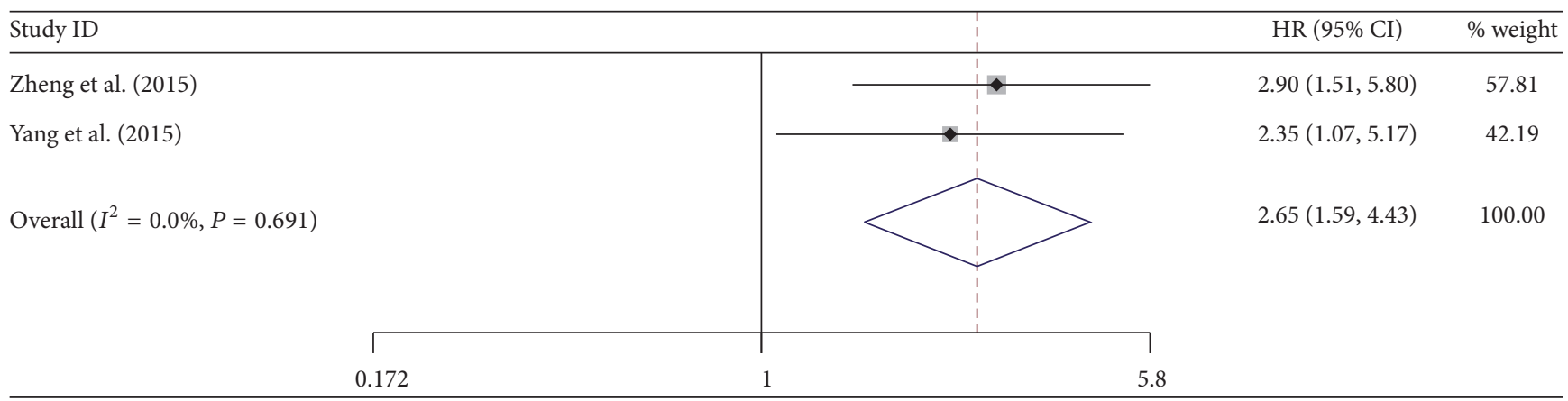

(a)

Study ID
Zheng et al. (2015)
Yang et al. (2015)
Shang et al. (2016)
Overall $\left(I^{2}=0.0 \%, P=0.992\right)$
0.169

(b)

FIGURE 4: Forest plots of hazard ratios (HRs) for the association between UCA1 expression with disease-free survival (DFS) from univariate analysis results (a) and DFS from multivariate analysis results (b).

above suggested that UCA1 plays an important role during the carcinogenesis and may serve as a potential target for treatment of digestive system malignancies. Recently, several studies have investigated the clinicopathological value of UCA1 expression in digestive system malignancies. However, the sample sizes in most studies are small. Besides, it is inconclusive about the association between UCA1 expression and progression of digestive system malignancies, such as metastasis and survival. Therefore, we conducted this metaanalysis with the aim of clarifying the clinical significance of UCA1 expression in digestive system malignancies.

To the best of our knowledge, this is the first meta-analysis to investigate the association of UCA1 expression with the metastasis and prognosis of digestive system malignancy patients. We included 10 studies with a total of 1089 patients. The pooled ORs with their 95\% CIs showed that high UCA1 expression was significantly associated with LNM and DM, indicating that UCA1 was an indicator for metastasis of digestive system malignancies. Moreover, the pooled HRs with their 95\% CIs showed that UCA1 overexpression was also significantly correlated with both poor OS and poor DFS, indicating that UCA1 overexpression may serve as an indicator of poor survival rate and high recurrence rate of digestive system malignancies, respectively. What is more, as the IncRNA can be secreted by cancer cells or released into the circulation from dead cancer cells, it has been reported that UCA1 level in plasma significantly decreased 14 days after surgery of colon cancer [19]. To some extent, it demonstrated the correlation between UCA1 overexpression and the aggressive behavior of digestive system malignancies, which was concordant with our conclusion. Taken together, UCA1 could serve as a promising biomarker for monitoring the progression of digestive system malignancies.

Nevertheless, there are several limitations in this metaanalysis. Firstly, most of the included studies were performed in the population from Asian countries rather than worldwide population; our results should be substantiated by additional studies in other races. Secondly, publication bias was observed in the studies with OS, which may be due to the fact that some studies were not included in this meta-analysis for their insufficient data or that some studies reported the correlation in one analytic method. Thirdly, the included studies were of small sample size as well as different cut-off value of UCA1 expression, which may generate errors by variation. Based on these limitations, the pooled ORs and HRs with their 95\% CIs calculated in this meta-analysis may be just estimations.

\section{Conclusion}

In conclusion, our meta-analysis showed that UCA1 overexpression was correlated with LNM, DM, poor OS, and 


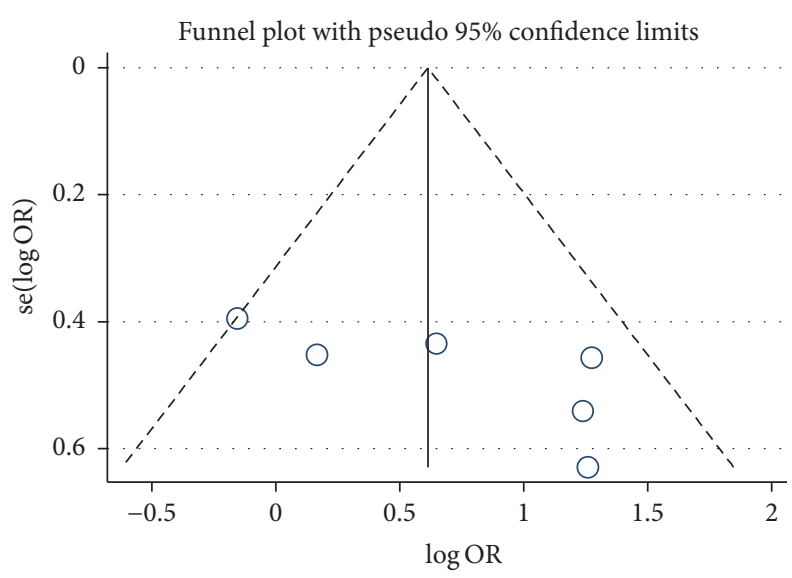

(a)

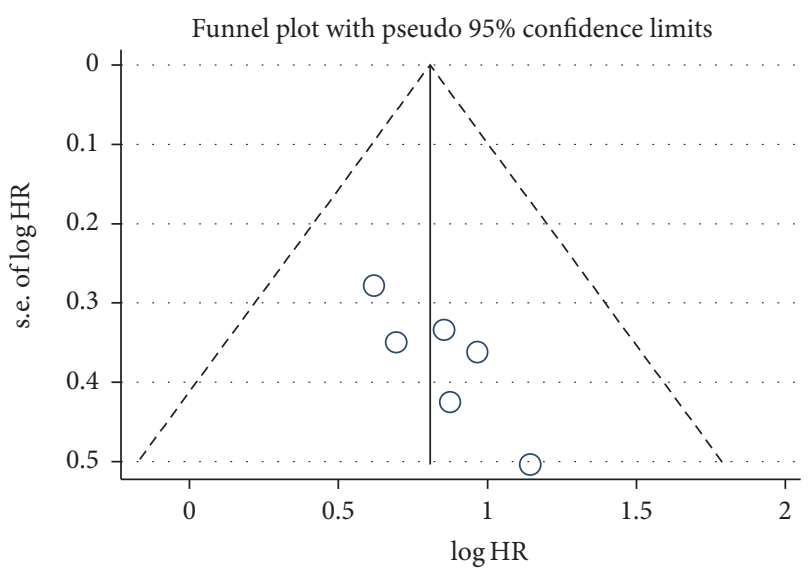

(c)

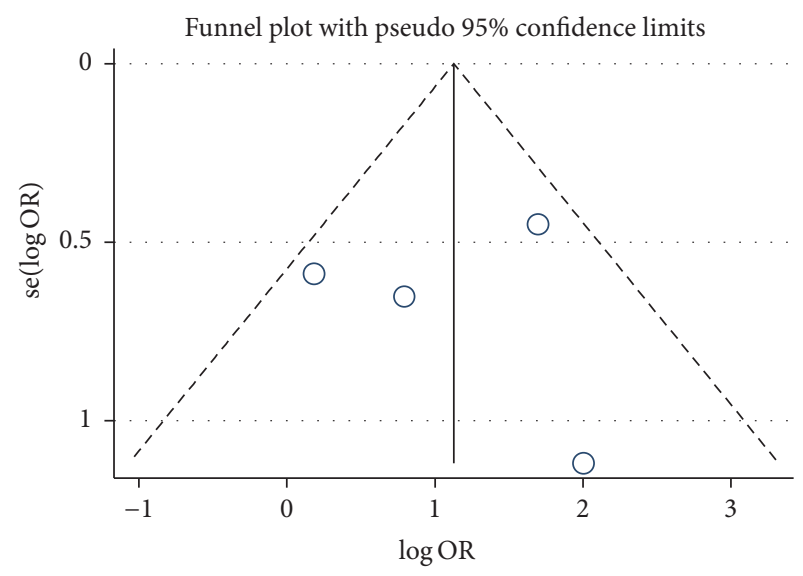

(b)

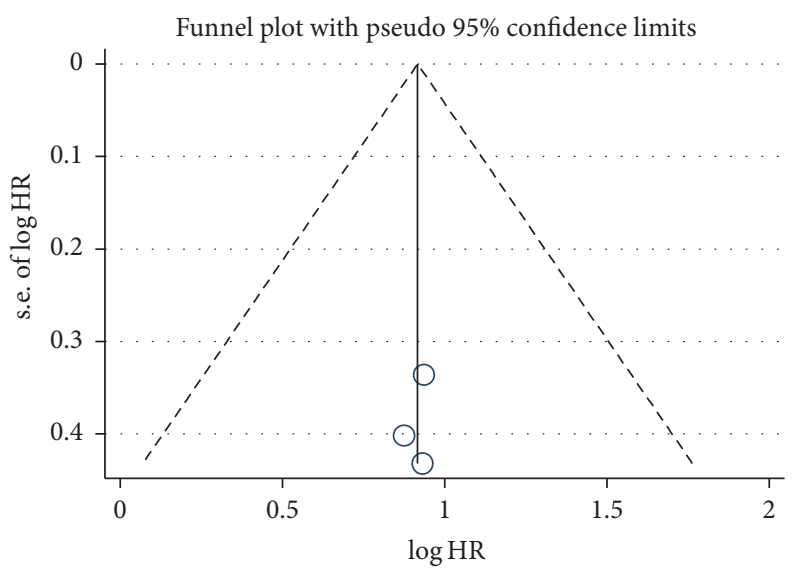

(d)

Figure 5: Funnel plots for the meta-analysis with lymph node metastasis (LNM) (a), distant metastasis (DM) (b), overall survival (OS) from multivariate analysis results (c), and disease-free survival (DFS) from multivariate analysis results (d).

poor DFS in digestive system malignancy patients. Therefore, UCA1 may serve as an indicator of metastasis and prognosis in digestive system malignancies.

\section{Competing Interests}

The authors declare that they have no competing interests.

\section{Authors' Contributions}

Xiao-Dong Sun and Chen Huan have contributed equally to this work.

\section{References}

[1] L. A. Torre, F. Bray, R. L. Siegel, J. Ferlay, J. Lortet-Tieulent, and A. Jemal, "Global cancer statistics, 2012," CA: A Cancer Journal for Clinicians, vol. 65, no. 2, pp. 87-108, 2015.

[2] Z. Cui, Y. Chen, Z. Xiao et al., "Long noncoding RNAs as auxiliary biomarkers for gastric cancer screening: a pooled analysis of individual studies," Oncotarget, vol. 7, no. 18, pp. 2579125800, 2016.
[3] J. Hou, Y. Zhou, Y. Zheng et al., "Hepatic RIG-I predicts survival and interferon-alpha therapeutic response in hepatocellular carcinoma," Cancer Cell, vol. 25, no. 1, pp. 49-63, 2014.

[4] P. Johnsson, L. Lipovich, D. Grandér, and K. V. Morris, "Evolutionary conservation of long non-coding RNAs; sequence, structure, function," Biochimica et Biophysica Acta (BBA)General Subjects, vol. 1840, no. 3, pp. 1063-1071, 2014.

[5] T. R. Mercer, M. E. Dinger, and J. S. Mattick, "Long non-coding RNAs: insights into functions," Nature Reviews Genetics, vol. 10, no. 3, pp. 155-159, 2009.

[6] J. Zhang, A. Zhang, Y. Wang et al., "New insights into the roles of ncRNA in the STAT3 pathway," Future Oncology, vol. 8, no. 6, pp. 723-730, 2012.

[7] H. Zhang, Z. Chen, X. Wang, Z. Huang, Z. He, and Y. Chen, "Long non-coding RNA: a new player in cancer," Journal of Hematology and Oncology, vol. 6, no. 1, article 37, 2013.

[8] S. Wang and Z. Wang, "Prognostic value of long noncoding RNA HOTAIR in digestive system malignancies," Journal of Gastroenterology and Hepatology, vol. 30, no. 7, pp. 1123-1133, 2015.

[9] H. Zhai, X.-M. Li, A. Maimaiti et al., "Prognostic value of long noncoding RNA MALAT1 in digestive system malignancies," International Journal of Clinical and Experimental Medicine, vol. 8, no. 10, pp. 18099-18106, 2015. 
[10] G. Ma, Q. Wang, C. Lv et al., "The prognostic significance of HOTAIR for predicting clinical outcome in patients with digestive system tumors," Journal of Cancer Research and Clinical Oncology, vol. 141, no. 12, pp. 2139-2145, 2015.

[11] F. Wang, X. Li, X. Xie, L. Zhao, and W. Chen, "UCA1, a nonprotein-coding RNA up-regulated in bladder carcinoma and embryo, influencing cell growth and promoting invasion," FEBS Letters, vol. 582, no. 13, pp. 1919-1927, 2008.

[12] Y. Fan, B. Shen, M. Tan et al., "Long non-coding RNA UCA1 increases chemoresistance of bladder cancer cells by regulating Wnt signaling," The FEBS Journal, vol. 281, no. 7, pp. 1750-1758, 2014.

[13] F. Wang, H.-Q. Ying, B.-S. He et al., "Upregulated lncRNAUCA1 contributes to progression of hepatocellular carcinoma through inhibition of miR-216b and activation of FGFR1/ERK signaling pathway," Oncotarget, vol. 6, no. 10, pp. 7899-7917, 2015.

[14] Z. Yang, Y. Lu, Q. Xu, B. Tang, C.-K. Park, and X. Chen, "HULC and H19 played different roles in overall and diseasefree survival from hepatocellular carcinoma after curative hepatectomy: a preliminary analysis from gene expression omnibus," Disease Markers, vol. 2015, Article ID 191029, 9 pages, 2015.

[15] Q. Zheng, F. Wu, W.-Y. Dai et al., "Aberrant expression of UCA1 in gastric cancer and its clinical significance," Clinical and Translational Oncology, vol. 17, no. 8, pp. 640-646, 2015.

[16] C. Shang, Y. Guo, J. Zhang, and B. Huang, "Silence of long noncoding RNA UCA1 inhibits malignant proliferation and chemotherapy resistance to adriamycin in gastric cancer," Cancer Chemotherapy and Pharmacology, vol. 77, no. 5, pp. 10611067, 2016.

[17] Y. Han, Y.-N. Yang, H.-H. Yuan et al., "UCA1, a long noncoding RNA up-regulated in colorectal cancer influences cell proliferation, apoptosis and cell cycle distribution," Pathology, vol. 46, no. 5, pp. 396-401, 2014.

[18] B. Ni, X. Yu, X. Guo et al., "Increased urothelial cancer associated 1 is associated with tumor proliferation and metastasis and predicts poor prognosis in colorectal cancer," International Journal of Oncology, vol. 47, no. 4, pp. 1329-1338, 2015.

[19] K. Tao, J. Yang, Y. Hu et al., "Clinical significance of urothelial carcinoma associated 1 in colon cancer," International Journal of Clinical and Experimental Medicine, vol. 8, no. 11, pp. 2185421860, 2015.

[20] Z. Bian, L. Jin, J. Zhang et al., "LncRNA-UCA1 enhances cell proliferation and 5-fluorouracil resistance in colorectal cancer by inhibiting miR-204-5p," Scientific Reports, vol. 6, article 23892, 2016.

[21] D.-T. Chen, A. H. Davis-Yadley, P.-Y. Huang et al., "Prognostic fifteen-gene signature for early stage pancreatic ductal adenocarcinoma," PLoS ONE, vol. 10, no. 8, article e0133562, 2015.

[22] J.-Y. Li, X. Ma, and C.-B. Zhang, "Overexpression of long noncoding RNA UCA1 predicts a poor prognosis in patients with esophageal squamous cell carcinoma," International Journal of Clinical and Experimental Pathology, vol. 7, no. 11, pp. 79387944, 2014.

[23] J. F. Tierney, L. A. Stewart, D. Ghersi, S. Burdett, and M. R. Sydes, "Practical methods for incorporating summary time-toevent data into meta-analysis," Trials, vol. 8, article 16, 2007.

[24] A. Stang, "Critical evaluation of the Newcastle-Ottawa scale for the assessment of the quality of nonrandomized studies in metaanalyses," European Journal of Epidemiology, vol. 25, no. 9, pp. 603-605, 2010. 


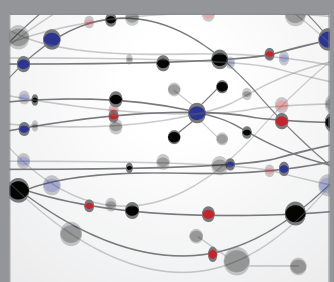

The Scientific World Journal
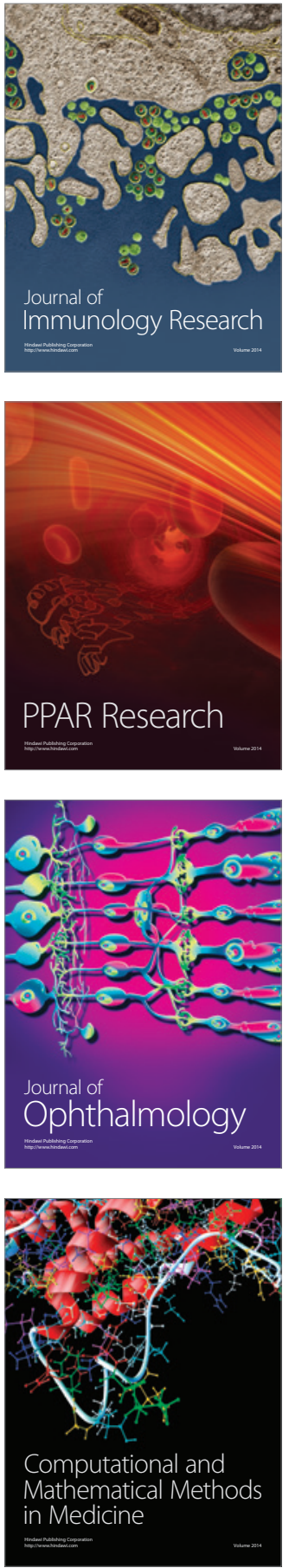

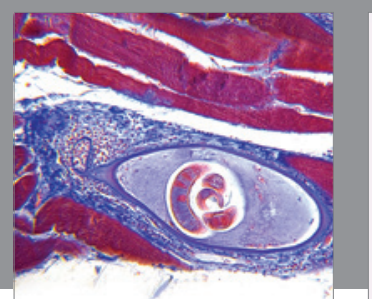

Gastroenterology Research and Practice

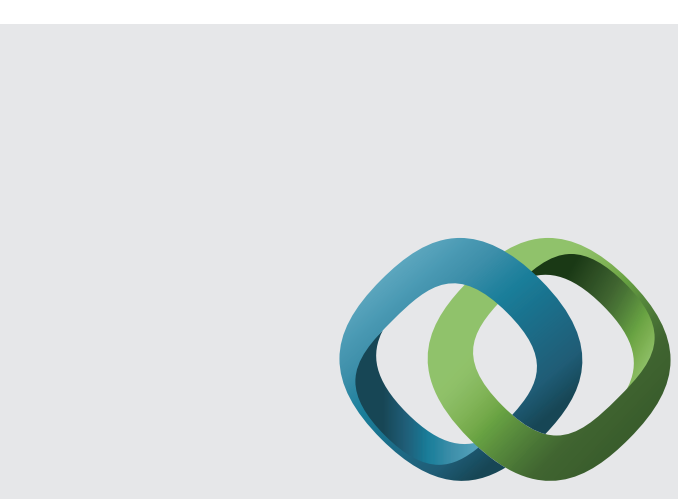

\section{Hindawi}

Submit your manuscripts at

http://www.hindawi.com
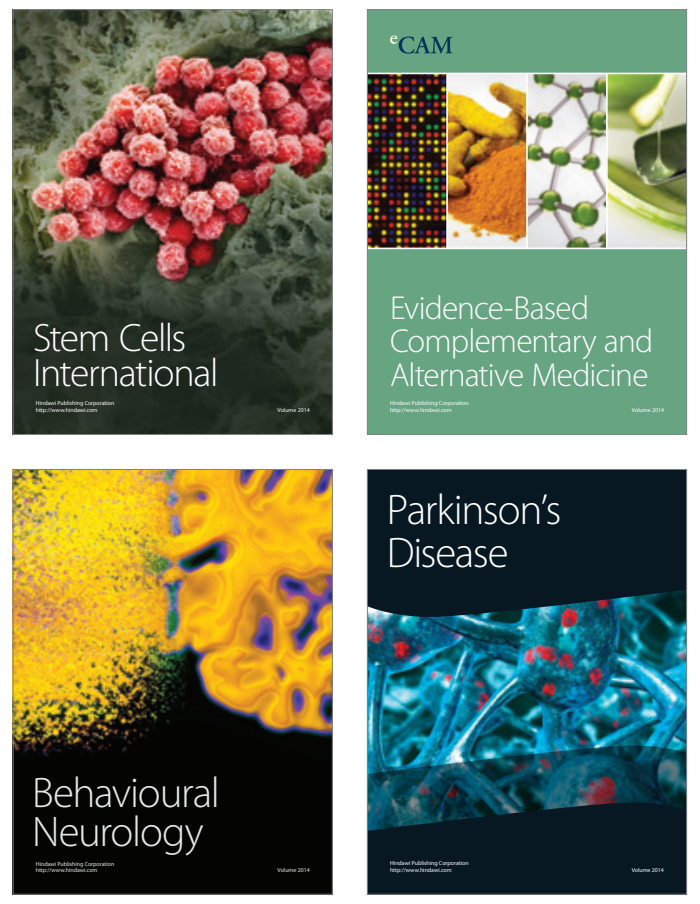
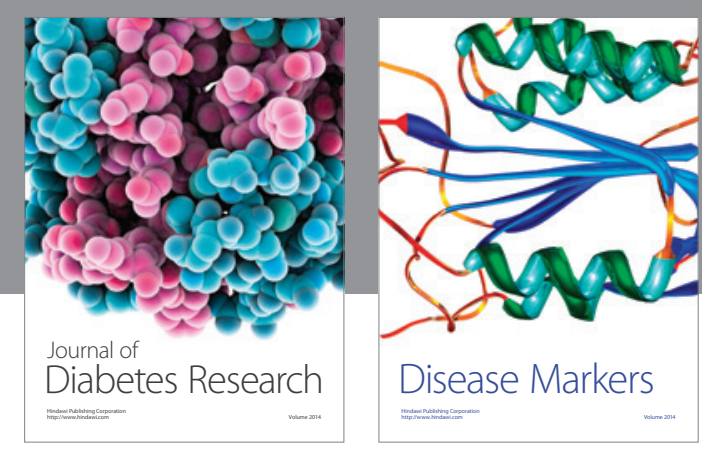

Disease Markers
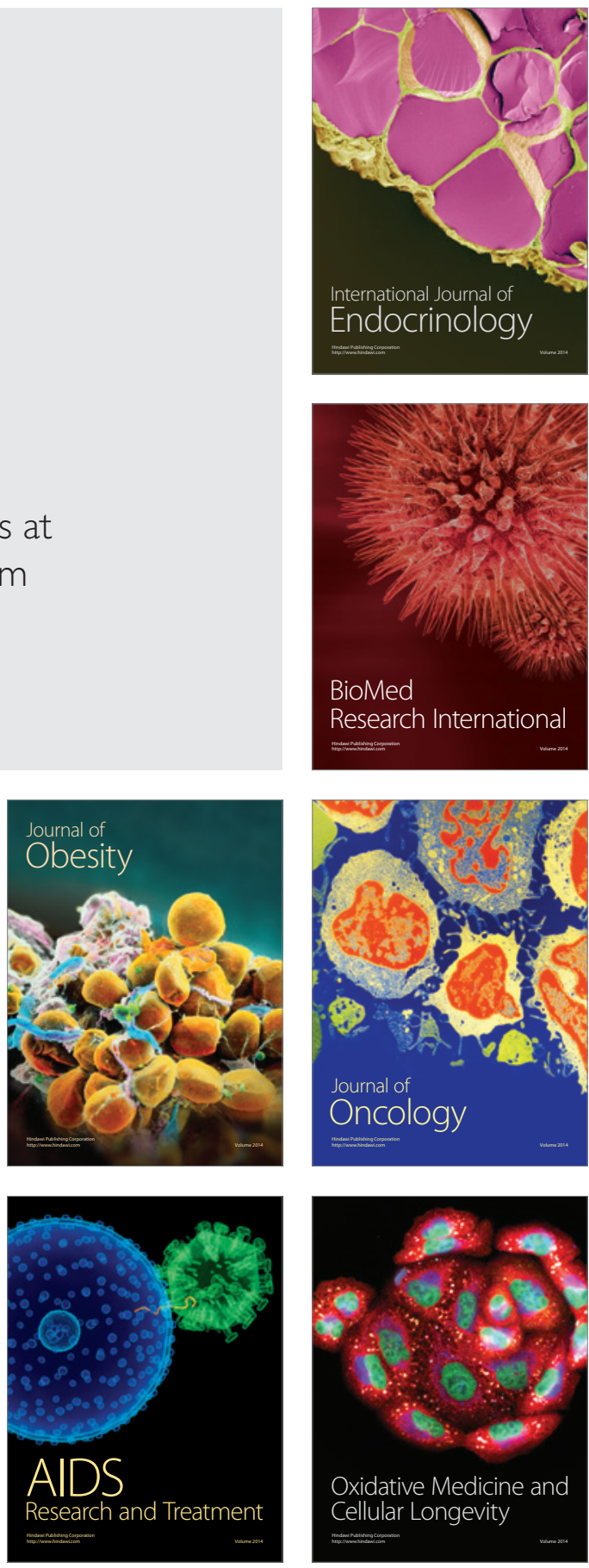